\title{
Paris-Murcie, París-Murcia, Murcia-París : la presse et la charité sans frontières, en France et en Espagne, en 1879
}

\author{
Jean-François BOTREL \\ Université Rennes 2 \\ botrel.j-f@orange.fr
}

\section{Résumé}

Cet article étudie le cas assez étonnant de trois journaux, dont un unique exemplaire a été publié à l'occasion des inondations qui eurent lieu à Murcie (Espagne) en octobre 1879, donnant à cet évènement une dimension internationale. Le Paris-Murcie, parisien, s'est doublé d'une réplique madrilène París-Murcia qui, elle-même, connaît une version murcienne (Murcia-París). Ces publications, essentiellement destinées à récolter des fonds pour les sinistrés, fournissent une illustration exceptionnelle de la manière dont les textes circulent d'une langue à une autre, d'un pays à un autre et d'un périodique à un autre.

Mots-clefs : Presse. Espagne. France. XIX ${ }^{e}$ siècle. Circulation des textes.

\begin{abstract}
This article examines the astonishing case of three newspapers, only one of each was published on the occasion of the floods that took place in Murcia (Spain) in October 1879, giving this event an international dimension. The Parisian Paris-Murcie was duplicated in Madrid as the Paris-Murcia which, itself, had a murcian version Murcia-Paris. These publications, mainly aimed at raising funds for the victims, provide an exceptional illustration of how texts circulate from one language to another, from one country to another and from one periodical to another.

Keywords: Press. Spain. France. 19th century. Circulation of texts.

\section{Resumen}

Este artículo examina el asombroso caso de tres periódicos, de los cuales solo un número fue publicado con motivo de las inundaciones que tuvieron lugar en Murcia (Espańa) en octubre de 1879, dando a este evento una dimensión internacional. El Paris-Murcie, parisino, dio lugar a una réplica madrileña (París-Murcia) que, a su vez, tuvo una versión murciana (Murcia-París). Estas publicaciones, destinadas principalmente a recaudar fondos
\end{abstract}

* Artículo recibido el 28/02/2019, aceptado el 28/10/2019. 
para las víctimas, constituyen una ilustración excepcional de cómo circulan los textos de un idioma a otro, de un país a otro y de un periódico a otro.

Palabras clave: Prensa. España. Francia. Siglo XIX. Circulación de textos.

L'Espagne blessée, la France saigne ; le coup qui frappe Murcie atteint Paris. Paris est la capitale du monde; et toute la douleur du monde est une douleur de Paris.

Victor Hugo (Paris-Murcie, p. 3)

\section{Introduction}

Pourquoi s'intéresser à ces trois titres qui n'ont, chacun, publié qu'un seul numéro et ne peuvent être classés dans la presse allophone de leurs pays respectifs, la France et l'Espagne?

Peut-être parce que, à propos d'un événement survenu en Espagne (les inondations de Murcie du 15 octobre 1879), il s'agit pour la presse française d'inverser la logique qui préside le plus souvent à la production d'une presse en langue étrangère à l'usage de lecteurs homophones, en donnant à un événement localisé, une dimension nationale et internationale, par la publication d'abord en français d'un numéro unique de Paris-Murcie, la publication quasi simultanée du même journal, en espagnol et à Madrid (Paris-Murcia), et d'une réplique murcienne (Murcia-París) permettant de conforter la dimension française et internationale de ce qui est présenté comme une entreprise de charité sans frontières. Avec pour résultats, les sommes collectées grâce à la vente des numéros, pour venir en aide aux "inondés de Murcie ", mais aussi une sorte de fusion confraternelle, linguistique et culturelle, francohispanique qui bien qu'éphémère mérite certainement d'être analysée ${ }^{1}$.

Rappelons les faits. Le 15 octobre 1879, une crue subite du Segura, dite Riada de Santa Teresa, ravage une partie de la région de Murcie, occasionnant 777 morts et des sinistrés sans nombre. La nouvelle, dont la une du Diario de Murcia se fait l'écho le lendemain, est immédiatement transmise dans le monde entier par des agences de presse comme Fabra ou Havas, et les principaux journaux français repren-

\footnotetext{
${ }^{1}$ Les études consacrées à l'inondation de Murcie d'octobre 1879 et à Paris-Murcie, ainsi qu'à la référence à la fête Paris-Murcie figurant dans $\grave{A}$ la recherche $d u$ temps perdu ou à Victor Hugo, sont listées à la fin de la présente étude qui doit beaucoup aux systèmes d'information offerts par le web. Cfr. : Arroyo Cabello (2018), Galderich (2009), Garre (s.d.), Hernández (1987-1989), Quiñonero (2014) et Valero (2015).
} 
nent bientôt des images transmises par des informateurs présents sur les lieux ${ }^{2}$, avant de dépêcher, pour certains d'entre eux, un envoyé spécial' ${ }^{3}$.

L'opinion française se trouve donc rapidement mobilisée autour de la nécessité de se montrer solidaire, en même temps que les Espagnols eux-mêmes ${ }^{4}$. L'inondation de Murcie devient une cause internationale, celle des «inondés de Murcie». Et c'est la presse parisienne qui va impulser ce mouvement de charité qui implique toute sorte d'acteurs.

\section{Paris-Murcie et l'Espagne}

Le Comité de la presse parisienne ${ }^{5}$, avec à sa tête le directeur de l'agence Havas, Édouard Lebey, après avoir envisagé d'organiser une course de taureaux à Paris, va opter pour la publication d'un numéro unique d'un journal illustré, finalement intitulé Paris-Murcie, qui recueillerait et publierait des contributions des personnalités de la politique, des arts et des lettres, de France et d'Espagne, ainsi que des autographes des souverains et dirigeants les plus illustres du reste du monde.

S'agissant de l'Espagne, on sait que Lebey se mit en relation avec l'agence de presse Fabra, et que, pour mener à bien le projet et recueillir des informations sur de potentiels collaborateurs espagnols, il s'appuya largement sur la colonie espagnole présente à Paris, aussi bien l'ancienne reine d'Espagne, Isabel de Bourbon, que le républicain en exil Manuel Ruiz Zorrilla, ou des journalistes et traducteurs.

${ }^{2} C f$., par exemple, L'Univers illustré du $1^{\text {er }}$ novembre 1879 ("L'inondation de la plaine de Murcie d'après un croquis de M. Fernando M. ") ou Le Monde Illustré qui entre le $1^{\text {er }}$ et le 15 novembre publie une quinzaine de dessins, certains sur une double page, à propos de ce qu'il appelle «les désastres de Murcie ». Sur ce traitement graphique de l'événement, voir l'étude de Martínez Jodar (2018).

${ }^{3}$ Comme M. Dick de L. qui adresse au Monde Illustré, assortis de commentaires, des croquis qui sont ensuite dessinés par Vierge ou Scott pour Le Gaulois où les articles d'Octave Mirbeau sont publiés les 6, 8 et 9 décembre 1879. "Le coup d'œil est effroyable, écrit-il. Plus un arbre debout, plus de pont sur la rivière. La terre est détrempée à un mètre de profondeur. La contrée a été comme dissoute et remaniée par le fléau... On dirait qu'un grand combat s'est délivré entre les éléments et que la terre a été vaincue. Partout des maisons renversées, des amas de ruines, des spectacles de destruction» (cité par le Dictionnaire Mirbeau, http://mirbeau.asso.fr/dicomirbeau [Consultation en ligne ; 9/10/2018].

${ }^{4}$ «Apenas hubo ciudad o pueblo grande o mediano (en incluso pequeño) ni gremio, compañía comercial, asociación o periódico que no se lanzase a la carrera de la solidaridad ", écrit Pedrosa (2005: 6), et les journaux publient régulièrement la liste des dons et donateurs. À l'initiative d'écrivains espagnols, un livre sera publié : (El) libro de la caridad dedicado por los poetas al socorro de las víctimas de las inundaciones en las provincias de Levante, 1879.

${ }^{5}$ Ce comité était composé de Lebey, Hippeau, rédacteur de l'Evènement et Montaigut, du même journal, Laffitte, directeur du Voltaire et Garnier, du même journal, Adrien Marx, rédacteur du Figaro et Arthur Meyer, directeur du Gaulois, et Levasseur du Globe, 
Le résultat de tous ces efforts dont la presse parisienne et espagnole rend fréquemment compte dans le courant des mois de novembre et de décembre ${ }^{6}$, est le numéro unique de Paris-Murcie, "journal publié au profit des victimes des inondations d'Espagne par le Comité de la presse française sous la direction de M. Édouard Lebey, directeur de l'Agence Havas, avec le concours de M. Lucien Marc, rédacteur en chef de l'Illustration et de M. E. Mercadier comme secrétaire de la rédaction » et qui porte la date de Décembre $1879^{7}$. Imprimé par E. Plon et Cie imprimeurs et éditeurs, il sera tiré à 150, 300 ou 400000 exemplaires - les chiffres varient selon les sources, mais le tirage est perçu comme «colossal " pour l'époque - et vendu 1 franc pour l'édition courante, 3 francs pour l'édition de luxe (en bristol) et même 20 francs pour quelques exemplaires en vélin. Il s'agit d'une publication de 24 pages $(40 \mathrm{x}$ $27 \mathrm{~cm}$ ), contenant 48 textes, 24 dessins et 50 autographes, ainsi que 34 annonces publicitaires.

Parmi les auteurs les plus connus des textes (articles, poèmes, maximes ou pensées, contes, lettres, etc.), on trouve Victor Hugo ( La Fraternité »), Zola (" De la douleur»), Alexandre Dumas, Daudet ("Souvenirs d'une inondation»), Emile Littré, Frédéric Mistral, François Coppée, Juliette Adam, mais aussi des académiciens comme Elme-Marie Caro et Octave Feuillet, et des représentants du monde du spectacle : Sarah Bernardht, Jacques Offenbach, ou la Patti (Adelina). Il y a trois représentants de l'Espagne, Manuel Ruiz Zorrilla, le marqués de Molins, ambassadeur d'Espagne en France, et le dramaturge et homme politique, Adelardo López de Ayala qui a adressé un sonnet, "El sol y la noche " - le seul texte en espagnol -, et mourra peu après, le 30 décembre.

Pour la plupart, ils ont adressé des textes déjà publiés par ailleurs et/ou peu en rapport avec l'Espagne. Les lecteurs de Paris-Murcie auront donc, sans doute, d'autant plus apprécié les articles originaux ou ceux qui témoignent d'une certaine connaissance de l'Espagne: le touchant poème en provençal de Frédéric Mistral, "Per l'Espagno ", daté de "Maïano, 11 nov. 1879 », celui de Déroulède consacré à "Murcie " (du temps d'Abd-el-Azir ben Bouça et Teodomir, en 710) ou l' "Amende honorable au Mançanarez " d'Alexandre Dumas, où celui-ci se souvient de ce que, il y a 33 ans, à Madrid lors d'une course de taureaux ayant bu quelques gorgées d'un verre d'eau fraîche, il rendit le verre au marchand d'eau en lui disant «Donnez le

\footnotetext{
${ }^{6}$ La presse espagnole suit avec attention la préparation de Paris-Murcie, comme en témoignent les fréquentes dépêches publiées dans El Imparcial, par exemple. Le 15 décembre 1879, l'Ilustración Española y Americana publie, p. 377, les portraits des «Individuos que componen el comité de prensa encargado de organizar la fiesta del hipódromo ».

${ }^{7}$ Une version électronique de Paris-Murcie est disponible sur Gallica ainsi que dans la Hemeroteca digital del Archivo municipal de Murcie.
} 
reste de ma part au Mançanarez » et il ne manque pas d'afficher sa connaissance de la langue espagnole, en s'exclamant, page 3 : « la Segura (quel nom trompeur !) vient de donner le plus éclatant et le plus terrible démenti à la plaisanterie que je fis alors ».

La proximité qu'a Murcie avec la Manche sert de prétexte à Elme-Marie Caro pour évoquer "Les Fils de Don Quichotte ». Edouard Laboulaye a adressé " Un vieux conte espagnol: Le mensonge et la vérité », Jacques Normand célèbre l'orange dans «La Bell’Valence!», Henri Martin les relations franco-espagnoles ("Espagne et France ») et le maréchal Canrobert, l'armée espagnole. Emile Littré, dans une lettre du 10 novembre 1879, fustige "l'abominable guerre" que Napoléon I ${ }^{\text {er }}$ fit à l'Espagne ». Quant à l'ambassadeur d'Espagne, le marqués de Molins, dans sa contribution intitulée "Paris-Murcie», il rappelle que "les journaux, les voyages, les échanges commerciaux, les pèlerinages, les expositions, les fêtes même, attachent bien plus fortement les nations entre elles, que les traités d'alliance et les pactes de famille».

Les dessins signés de Gustave Doré, Gérôme, Fantin-Latour, Meissonier, Madrazo et 19 autres, ne sont guère plus originaux ou pertinents. Seuls ceux de Doré, Louis Leloir, et A. de Neuville semblent avoir été réalisés ex-profeso. Sur la com-

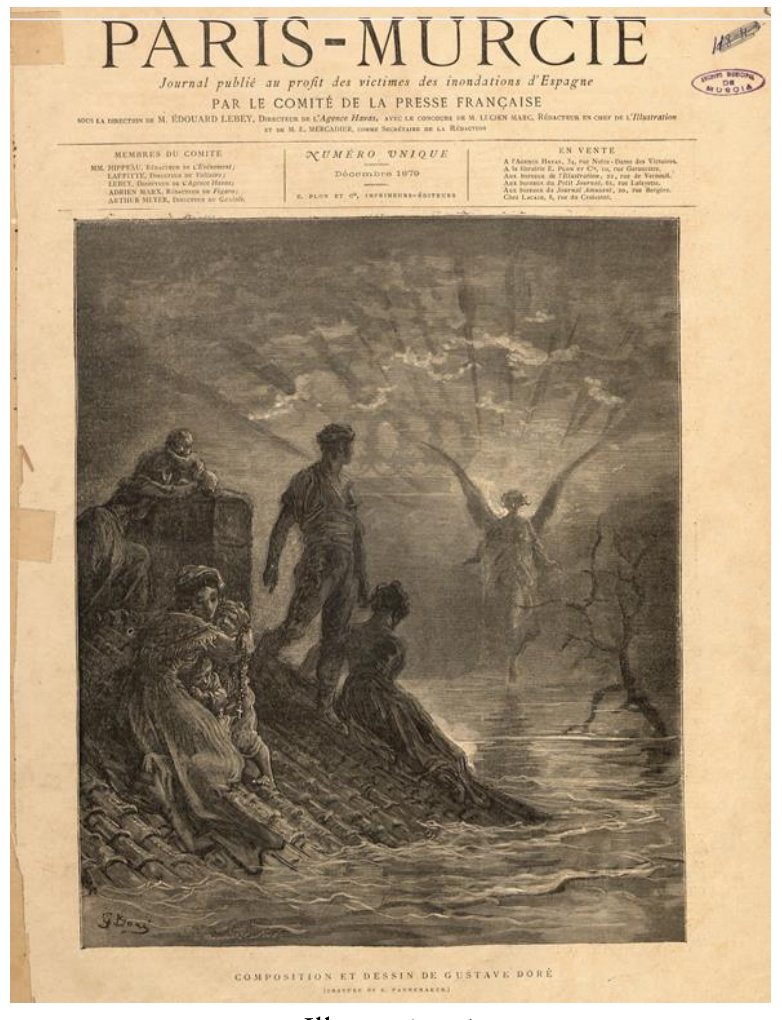

Illustration 1 position et le dessin de Meissonier, gravé par Ch. Baude, daté de 1879 et publié en hors-texte figurent bien des armes de Murcie approximatives, mais le héraut qui les arbore à sa trompette ressemble davantage à un cavalier Louis XIII, et le dessin de Georges Clairin, lu par El Imparcial du 23 décembre comme un "paysage de Murcie", fait plutôt penser au Maroc. Seule la composition de G. Doré qui figure sur la couverture de ParisMurcie est explicitement allusive. Voici la description qu'en fait, en espagnol, El Imparcial du 23 décembre: «Representa una escena de inundación: una casa rodeada de agua: sobre el tejado se ve refugiada la familia, grupos de madres abrazando a sus hijos y un huertano robusto y erguido mirando hacia oriente de donde sale el ángel de la esperanza por 
una rompiente de luz. Conjunto hermosamente sombrío". Le chapelet que porte bien en évidence la femme sur la gauche n'est pas mentionné. On peut évidemment se demander si, en l'occurrence, l'originalité et la pertinence ont beaucoup d'importance ? Comme le souligne à l'époque la Revue Bordelaise, dans son numéro 32, l'essentiel est que Paris-Murcie soit "signé des plus grands noms parmi ceux qu'il est convenu d'admirer ${ }^{8} "$.

Parmi les 50 autographes recueillis par l'entremise des ambassades, figurent des personnalités du monde entier (Bulgarie, Egypte Suisse, Turquie, Angleterre, Espagne, Pays-Bas, Danemark, Roumanie, etc.). La plupart d'entre elles écrivent en français, qui est encore à l'époque la langue de la diplomatie. C'est, par exemple, le cas des Espagnols, le roi Alphonse XII compris. Mais le Pape s'est exprimé en latin, les feld-maréchaux de Moltke et Manteuffel en allemand, Osman Pacha en turc, Lord Lyons et Gladstone en anglais, Garibaldi en italien. Cette relative diversité des nationalités et des langues autour du français dominant renforce évidemment la dimension cosmopolite d'une publication qui a vocation à être diffusée en dehors de France et à affirmer ainsi, à l'occasion d'une ouvre de charité, le statut de Paris comme «capitale du monde", pour reprendre l'expression de V. Hugo. Comme dans la plupart des journaux à l'époque, les dernières pages sont consacrées à la publicité : trois pages $\mathrm{d}$ 'annonces où les parfumeurs français occupent une belle place.

Paris-Murcie: entre ces deux entités, il y a tout un monde... Paris est alors perçue comme la capitale de l'Europe, la ville étrangère cosmopolite et emblématique de la civilisation européenne, par antonomase; Murcie (92 000 habitants) capitale d'une lointaine province espagnole est alors inconnue de la plupart des Français qui ont encore de l'Espagne une vision largement influencée par Dumas ou Gautier ou la Carmen de Bizet. Mais dans la formulation, Paris et Murcie se retrouvent mises sur le même plan et symboliquement et étroitement réunies par un trait d'union. Seul compte, dans les trois cas, la langue et la graphie utilisées et l'ordre des termes : ParisMurcie, París-Murcia ou Murcia-París.

Le relais assuré par la presse espagnole de l'initiative de la presse parisienne aura pour conséquences qu'avant même sa parution, le Paris-Murcie soit acheté, en Espagne, pour les archives et bibliothèques et que plus de 50000 exemplaires soit

\footnotetext{
${ }^{8}$ Cet élan de solidarité n'aura pas cependant emporté tous les «grands noms » : si Fantin-Latour apprécie l'écho que Paris-Murcie a donné à sa contribution, Gustave Flaubert se refuse à ce que les inondés "même de Murcie » lui fassent «faire et faire signer des turpitudes " (lettre à Emile Bergerat du 13 décembre 1879). De même il refusera de faire partie du Comité organisateur de la Fête du 18 décembre pour des raisons qu'il explique à sa correspondante, la princesse Mathilde : "la gent de lettre parisienne a l'air entortillée par les «inondés de Murcie "... La fête se passera de ma présence ne sachant ni danser le boléro ni pincer de la guitare».
} 
commandés en province9. Le vrai lancement de la vente de ce "numéro unique, né d'une pensée sublime", comme l'écrit la Revue Bordelaise, coïncide avec un autre événement parisien, également organisé par le Comité de la presse à des fins de chari-

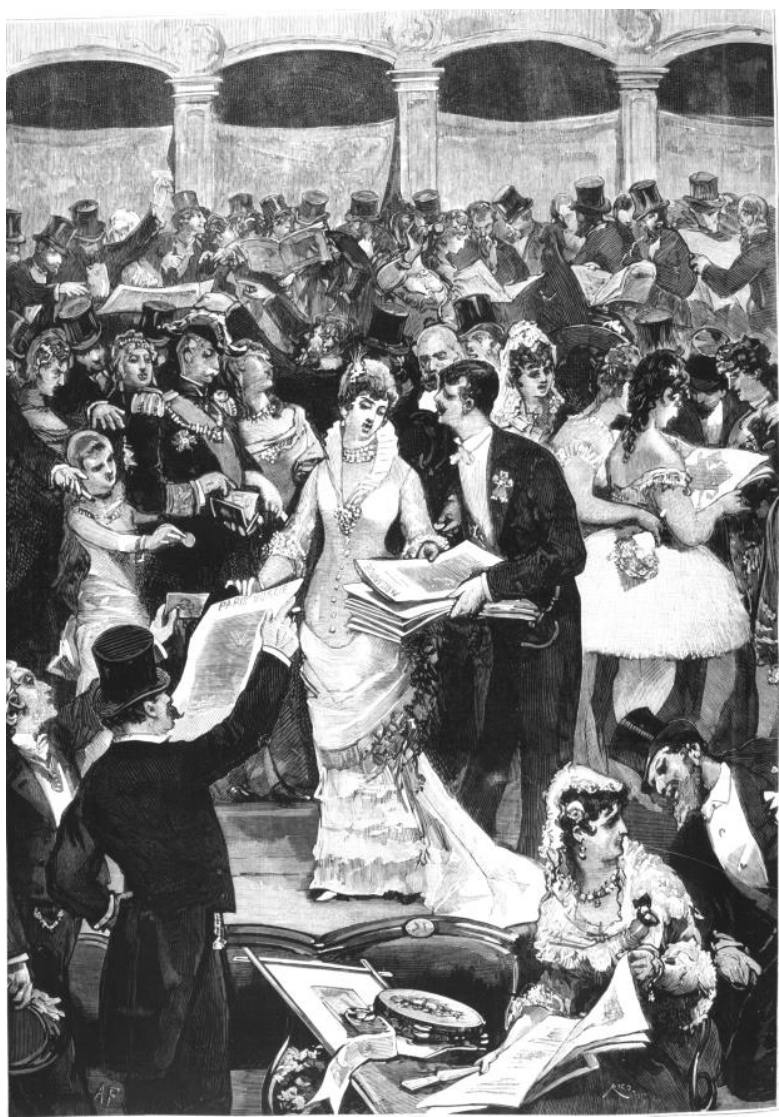
té, le 18 décembre, dans la salle de l'Hippodrome près du pont de l'Alma : une fête dite de Murcie, de l'Hippodrome, de ParisMurcie, de la presse, ou, selon Le Gaulois, des malheureux de France et d'Espagne, qui va être beaucoup plus «espagnole» que le journal. Près de dix mille spectateurs, "plus avides de charité que de plaisir» selon

${ }^{9}$ Cf. El Imparcial, des 4 et 12 décembre 1879. 
Illustration 2

Le Gaulois, vont se rendre à cet événement mondain ${ }^{10}$, dont on retiendra ici que le langage employé dans son ordonnancement est, essentiellement, à la fois allochtone et allophone, puisqu'il s'agit de s'adresser visuellement et, pour partie, linguistiquement, "en espagnol ", à un public majoritairement français, en plein cœur de Paris, mais dans un espagnol correspondant aux représentations dominantes en France.

En effet, pour cette fête, outre la présence des deux cents musiciens de l'orchestre dirigé par Olivier Mettra, de seize pianos, du corps de ballet de l'Opéra, de ventes aux enchères et de quelques élégantes à éventail et mantille, on a transformé l'arène ovale de l'Hippodrome en une "véritable ville espagnole " en carton, avec ses posadas et ses tiendas (celle du Monde Illustré, par exemple ${ }^{11}$ ). Là, domine une réduction de la tour de la Giralda, pourvue de trente cloches, avec, à côté, un pont censé rappeler celui du Segura, une réplique de l'Hospicio de Santo Domingo, des constructions de la huerta, une maison de Murcie avec « ses palmiers nains, ses plantations de noyers et ses éventails d'agaves". Sur un plancher de $4000 \mathrm{~m}^{2}$ défilent dans l'ordre: vingt-cinq guardias civiles, la musique de l'artillerie et du génie espagnol, puis, sur l'air de la grande Marche aux flambeaux (de Meyerbeer sans doute) suivi du pasodoble de Pepe Hillo, six alguaciles à cheval, des toreros ou espadas en vue à l'époque (Lagartijo, el Gordito, Gonzalo Mora, Pastor ${ }^{12}$ ), huit picadors à cheval, seize banderilleros, des mulilleros avec leurs six mules, suivis d'un orchestre de vingt-trois guitaristes en costume andalou, de cantaores et cantaoras et de danseurs gitans

\footnotetext{
${ }^{10} C f$. https://lefoudeproust.fr/2018/01/une-fete-pour-les-inondes-de-paris.

${ }^{11}$ Cf. Le Monde illustré du 27 décembre 1879, p. 409.

${ }^{12}$ Cf. Le Monde Illustré du 27 décembre 1879, pp. 412-413.
} 
qui se produiront sur scène ${ }^{13}$. On a pu aussi entendre l'invitation à la valse de Weber, la marche de Tannhauser, la symphonie de Guillaume Tell, la marche ou la symphonie d'Obéron - les dénominations des journalistes peuvent être hésitantes - la

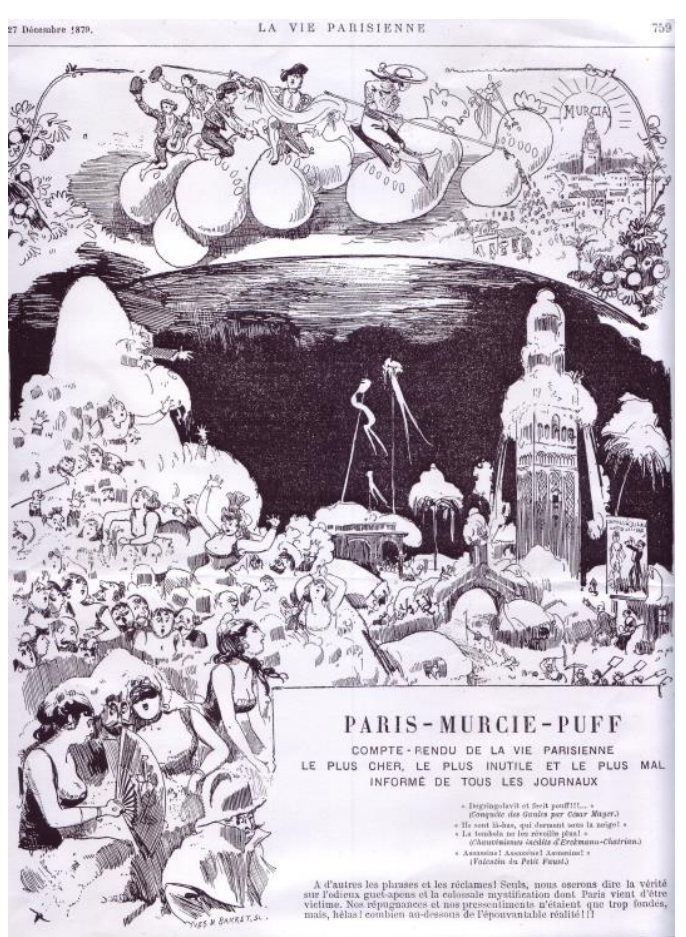

Illustration 3 .

Marche hongroise de Kowalski, l'ouverture de la Muette de Portici de Auber, etc. et deux ensembles anglais et autrichien, la volonté manifeste des organisateurs étant de donner à cette fête un caractère plus que simplement francoespagnol, international, sans pour autant en garantir totalement la cohérence. Puis, le carillon de la Giralda sonne le début de la verbena au cours de laquelle l'on dit la bonne aventure et l'on met aux enchères des panderetas (tambours de basque) décorées par les artistes, avant que ne s'ébranle une grande farandole.

Comme le dit El Liberal, cette fête « trasladaba enteramente la imaginación a cuanto de animado, bullicioso, alegre y placentero tiene esa España de pandereta y la manolería con que sueñan todos los franceses ".

Cette espagnolade, justement brocardée par Pedrosa (2015), mais qui remarquons-le - n'a pu être organisée qu'en étroite collaboration avec des Espagnols, a trouvé de larges échos dans la presse ${ }^{14}$. Elle coïncida avec une grande vague de froid peu conforme avec l'idée qu'on se fait du climat espagnol. C'est ce qui explique que La Vie Parisienne, qui dans son $n^{\circ}$ extraordinaire de 20 pages du 27 décembre (ParisMurcie-Puff), donne une vision décapante de la manifestation, n'hésite pas à parler de "la retraite de Murcie». Elle inclut également dix illustrations (dont une double page) signées d’Yves \& Barret, assorties de légendes en un très bon espagnol. On re-

${ }^{13}$ La majorquine Rosita Mauri (1850-1923), engagée en 1878, sur la recommandation de Charles Gounod, par l'Opéra de Paris, danse avec le corps de ballet de l'Opéra, et c'est pour elle que le torero Lagartijo « décroche vivement sa cape couleur lilas et la déploie sous ses pieds ». Olé !!

${ }^{14}$ Mais aussi chez certains écrivains, comme Marcel Proust ("c'était le jour de la fête de Paris-Murcie donnée pour les inondés de Murcie ", écrit-il dans $A$ la recherche du temps perdu) et, négativement, chez Octave Mirbeau (dans L'Ecuyère) ou chez Maupassant qui, dans Le Gaulois du 8 février 1882, dénonce, à propos des œuvres de bienfaisance envers l'étranger, "les saltimbanques qui battent la grosse caisse sur la peau des victimes ». 
marquera toutefois, sans pour autant conclure à l'appropriation de stéréotypes externes, que la presse madrilène s'est montrée beaucoup moins sévère, à en juger par les articles reproduits par Pedrosa (2015 : 15-29) ou la Ilustración Española y Americana ${ }^{15}$.

Le produit de cette fête s'élève à 600000 francs (300 000 selon Le Gaulois) qui s'ajoutent à celui correspondant à la vente de Paris-Murcie : on cite la somme de 1812000 pesetas remises à Murcie. Mais la mobilisation internationale ne s'arrête pas là, puisque, presque en même temps que la publication de Paris-Murcie, paraît à Madrid une traduction en espagnol du journal : París-Murcia ${ }^{16}$.

\section{París-Murcia ou la version espagnole}

On trouve dans El Imparcial du 12 décembre 1879, une explication à cette initiative. Selon le journal madrilène, journal de référence à l'époque :

el convencimiento de que son muchas las personas que se ven privadas de poder apreciar el mérito del periódico ParisMurcia, y el deseo de que todos los españoles satisfagan el gusto de admirar esta magnífica obra [...] nos han movido a solicitar del Comité de la prensa francesa, y hemos obtenido, la publicación en París de una edición en castellano con los mismos grabados, autógrafos y demás que contiene la francesa ».

Plus que les textes ce sont, on le voit, les dessins et les autographes qui importent et symboliquement la traduction en espagnol de Paris-Murcie permet à la presse madrilène de se montrer l'égale de la presse parisienne, en faisant transposer l'intégralité de la publication française en espagnol, pour permettre l'appropriation par des sphères plus larges. Comme si París-Murcia avait été publié en espagnol à Paris, mais l'impression sera finalement faite à Madrid. Dans ce cas aussi, on observe une circulation du journal dans une autre sphère linguistique, puisque le París-

\footnotetext{
15 «Interior del hipódromo en la noche del festival de la prensa » (cf. Fundación Joaquín Díaz; https://funjdiaz.net/ilustracion).

${ }^{16}$ París-Murcia, periódico publicado en francés por el Comité de la Prensa Francesa a beneficio de las víctimas de las inundaciones de España bajo la dirección de Don Eduardo Lebey, director de la Agencia Havas y la colaboración de don Luciano Marc, redactor en jefe de la Illustration y de don E. Mercadier, como secretario de redacción. Traducido al español por los periodistas españoles residentes en París, don Albert Araus, don Juan B(autista). Enseñat y don Angel Muro. Casa editorial de D. Jesús Otero Fernández, Concepción Gerónima 32 y calle del Arenal 37. París-Murcia peut être consulté à la Biblioteca Nacional de España (cote Z/3371; il s'agit de l'exemplaire de dépôt légal signé de Jesús Otero) et en ligne, comme "Traducción al español de Paris - Murcie» sur la page web http://www.regmurcia.com. El Imparcial qui annonce sa parution les 17 et 23 décembre, le qualifie de "periódico de caridad» et donne la liste des lieux où l'on peut l'acheter à Madrid. Prix de vente : 6 réaux pour l'édition courante et 12 pour l'édition de luxe sur papier bristol.
} 
Murcia se vend également à Paris (chez Jean Oller, 23 rue de la Boétie) et que le 21 décembre, El Imparcial informe ses lecteurs de ce que les exemplaires de París-Murcia commandés avant le 17 sont bien arrivés à Paris...

Il s'agit de la traduction presque intégrale de la version en français et on ne manque pas de souligner les difficultés qu'ont dû rencontrer les traducteurs " en la traducción al castellano de frases, modismos y vocablos franceses difíciles de interpretar aun por aquellos que poseen el idioma francés pero que no están familiarizados con las costumbres y corruptelas del lenguaje parisiense ». S'agissant des autographes, ils sont bien sûr reproduits à l'identique et transcrits en espagnol sur une feuille intercalaire. Parmi les trente-quatre annonceurs, on trouve la maison Guerlain, parfumeurs de la rue de la Paix, "dont les vives sympathies pour tout ce qui est espagnol sont connues et qui compte certainement autant de jolies clientes en Espagne qu'en France ", Violet parfumeur à Paris, fournisseur breveté de la Cour d'Espagne, «la haute société espagnole a été une des premières à patronner et partager les préparations de la maison Violet savon Royal de Thrédace, Veloutine, crème Pompadour, parfumerie au Champaka ", est-il précisé.

Alors qu'on ne dispose pas d'informations sur la diffusion et l'impact de Paris-Murcia, comment interpréter cette initiative? Sans doute la doit-on a la volonté d'amplifier le mouvement de solidarité. On ne peut, cependant, s'empêcher de penser que dans cette réplique en espagnol d'un journal français en Espagne, à l'initiative de la presse madrilène, il y a plus que la réalisation d'une traduction supplémentaire, pour permettre l'appropriation d'un bien en langue étrangère. Il y a sans doute le souhait de ne pas être en reste, de se montrer symboliquement à la hauteur de Paris et de permettre à un public hispanophone (l'exportation en Amérique latine n'est évidemment pas à exclure) de posséder et consommer un de ces nombreux " articles de Paris ", si demandés à l'époque: comme le dit El Imparcial, "al comprar ParísMurcia no solo hacen buena obra, sino enriquecen su biblioteca con una colección sin precedente y sin continuación en los fastos del periodismo ».

De toute évidence, le fait qu'à côté de toutes les initiatives charitables prises en Espagne, une telle initiative ait pu être prise à Paris qui pour l'Espagne est alors encore emblématique de l'Europe est particulièrement appréciée : la presse espagnole s'en fait largement l'écho et manifeste sa gratitude en même temps que sa fierté. C'est ainsi qu'on peut interpréter la réponse de la presse murcienne à l'initiative de la presse parisienne ${ }^{17}$, avec la publication le 18 décembre, d'un numéro également unique du

${ }_{17}$ A l'instar du Comité de la presse parisienne, il existait en Espagne une Junta Central de Socorros créée par la presse murcienne et madrilène (El Imparcial, El Correo, El Globo, El Liberal) et présidée par le directeur du Semanario Murciano. 
Murcia-París, la veille d'une manifestation solennelle de reconnaissance à la France, à Murcie.

\section{Murcia-París}

Il s'agit d'un huit pages in folio, imprimé à Murcie (Tip. H. de Nogués) et sans illustrations, vendu 50 centimes de peseta, et dédié "A la prensa de París, testimonio de admiración y agradecimiento " ou comme le dit l'un des contributeurs, pour répondre «modesta, pero dignamente al PARÍs-MURCIA que la prensa francesa dedica al socorro de nuestra catástrofe ". Au delà des collaborations qu'il contient, qui toutes sauf une (celle de José Echegaray) sont l'œuvre de plumes murciennes et visent presque toutes, sous des formes diverses - il y a beaucoup de poèmes - à manifester la gratitude de Murcie à l'égard de la France ${ }^{18}$, le Murcia-París dit explicitement combien les murciens ont pu se sentir flatté de ce que, comme l'écrit (p. 5), Felipe Blanco de Ibáñez, «el emporio de la civilización » ait pleuré avec eux. Et Rodolfo Cárles, page 8 , ne dit pas autre chose: « un honor y muy distinguido para Murcia es ver su nombre asociado al de la gran capital».

L'événement (les inondations de Murcie mais plus encore la publication du Paris-Murcie) a donc pris une dimension transnationale et inspiré aussi, comme souvent à l'époque, toute sorte de créations dramatiques d'actualité, en Espagne et en France. Mais il est, sans doute, encore plus intéressant de constater que c'est dans le langage plus universel de la musique que l'impact de l'initiative du Comité de la presse s'est le plus manifesté : on ne compte pas moins de sept morceaux intitulés Paris-Murcie ou París-Murcia, composés par des Espagnol(e)s ou des Français(es) en 1880.

Juan González Castaño et Ginés J. Martín-Consuegra (2017 : 140) font référence à un numéro spécial du journal satirique, républicain et anticlérical barcelonais La Campana, publié le 29 janvier 1880, au bénéfice des victimes de l'inondation conservatrice qui vient de se produire ("Á benefici de las víctimas de la inundació conservadora ») ainsi qu'à un pliego de aleluyas (image d'Epinal) intitulé Inundación de Murcia, également imprimé à Barcelone.

\section{4. «Il n'y a plus de Pyrénées"}

À travers Paris-Murcie, París-Murcia et Murcia-París, au delà des résultats financiers et d'un mimétisme assez fréquent à l'époque, il y a certainement aussi la satisfaction de pouvoir partager, au delà des frontières, un esprit de solidarité et de bienfaisance. En décembre 1879, on peut sans doute croire Albert Wolff quand il

\footnotetext{
${ }^{18}$ Le détail des contributions est donné dans El Imparcial du 23 décembre et dans l'article de Juan Garre (s.d.).
} 
assure, dans un article intitulé "Il n'y a plus de Pyrénées ", publié dans Paris-Murcie, que « la ville de Murcie est située dans une province espagnole du grand Empire idéal de la Charité, pour lequel il n'y a ni frontières, ni différence de races, où tous les habitants parlent la même langue, celle du cœur, et où tous les citoyens sont égaux devant la solidarité humaine». Tant l'original français que sa traduction espagnole ou que la modeste réplique murcienne rendent, en effet, compte d'une internationalisation de l'information (agences, reporters, circulation des images) mais aussi de la charité et Murcia-París peut saluer, page 4, l'« explosión caritativa de la prensa de París, producida por nuestros desastres, cual chispazo eléctrico comunicada a toda $\mathrm{pa}^{19} »$.

Bien avant le phénomène "Je suis Charlie " en 2015, on observe, en effet, autour des «inondés de Murcie », le dépassement des frontières nationales et linguistiques et une sorte d'emballement général, favorisé à l'époque par les agences de presse et la presse elle-même qui, profitant de l'aura dont bénéficie Paris, favorise la circulation de journaux en français ou en espagnol publiés pour répondre, par des actes de bienfaisance, à une catastrophe survenue à Murcie.

Une première "globalisation " qui contraste assez fortement avec la démarche ethno-centrée ou communautaire qui est parfois associée à la presse allophone.

\section{RÉFÉRENCES BIBLIOGRAPHIQUES}

Arroyo Cabello, María (2018) : «La riada de 1879 en El Diario de Murcia. Una campaña de prensa con repercusión internacional ». Revista Portuguesa de História da Comunicação, 2. [Consultation en ligne: http://www.revistahc.sopcom.pt/ficheiros/20180130-mar_a_arroyo_cabello.pdf; 29/9/2018].

GARRE, Juan Antonio (s.d.) : «Paris-Murcie un periódico para la historia ». Región de Murcia digital». [Consultation en ligne: https://www.regmurcia.com/servlet/s.Sl?sit=c,373,m,1096\&rr=ReP-24530-DETALLE_REPORTAJESPADRE ; 15/9/2018].

GALDERICH (2009): "Paris-Murcie del 1879 (I), la primera iniciativa solidària internacional ?». Piscolabis librorum, 12 octobre [Consultation en ligne: http://librorum.piscolabis.cat/2009/10/paris-murcie-del-1879-i-la-primera.html ; 5/12/2016].

GONZÁlEZ CASTAÑO, Juan et Ginés J. MARTíN-CONSUEGRA BLAYA (2017) : El grabado en Murcia, siglos XVII-XIX. Murcia, Real Academia Alfonso X el Sabio.

HERnÁNDEZ, Francisco Javier (1987-89) : «Ecos de la Fiesta París-Murcia en La Recherche du temps perdu ». Estudios románicos, 4, 563-568.

${ }^{19} \mathrm{~L}$ '« explosion charitable de la presse de Paris, produite par nos désastres, telle une étincelle électrique communiquée à toute l'Europe ". 
MARTÍNEZ JÓDAR, Manuel (2018) : « Fotografía y catástrofes en la España del siglo XIX : el reportaje sobre las inundaciones en Murcia en octubre de 1879 ", in J. A. Hernández Latas (ed.), II jornadas de investigación en historia de la fotografía. 1839-1939: un siglo de fotografía. Zaragoza, Instituto Fernando el Católico, 47-57 [Consultation en ligne : https://ifc.dpz.es/recursos/publicaciones/37/13/04martinezjodar.pdf; $7 / 12 / 2018]$.

MOLINÉ, Jean (2015) : «La riada del Segura en 1879 y la fiesta París-Murcia o la desgracia de unos provoca la felicidad de otros". Tonos digital. Revista de Estudios Filológicos, 28. [Consultation en ligne: http://www.um.es/tonosdigital/znum28/secciones/relecturas-3--la_riada_del_segura.htm ; 8/10/2018]

Pedrosa, José Manuel (2015) : «La fiesta española de L'Hippodrome de París (18 de diciembre de 1879) : toros, flamenco y seudo folclore español ». Boletín de Literatura Oral, 5, 9-33.

QUiÑONERO, Juan Pedro (2014) : "París y Murcia unidas por Victor Hugo ». Una temporada en el infierno, 4 mars. [Consultation en ligne : http://unatemporadaenelinfierno.net/2014/03/04/paris-y-murcia-unidas-por-victor-hugo ; 8/9/2018]

SOTELo, Marisa (2003) : «Un inédito de Emilia Pardo Bazán con finalidad solidaria : breves notas de sociología literaria. La Tribuna, 1, 149-162.

Valero, Francisco (2015) : «La deuda de Murcia con París». La Opinión de Murcia, 16 novembre. [Consultation en ligne: https://www.laopiniondemurcia.es/comunidad/2015/11/16/deuda-murcia-paris/692586.html ; 8/9/2018]

\section{ILLUSTRATIONS}

Illustration 1 : Composition de Gustave Doré (couverture de Paris-Murcie, décembre 1879; Gallica).

Illustration 2: «La venta del París-Murcia en las galerías del hipódromo, por la Comisión de actrices » (La Ilustración Española y Americana, 31 décembre 1879, 416.

Illustration 3 : La Vie Parisienne, n 52, samedi 27 décembre 1879, p. 759 (col. JFB).

Para citar este artículo / Pour citer cet article :

Botrel, Jean-François (2019): «Paris-Murcie, París-Murcia, Murcia-París: la presse et la charité sans frontières, en France et en Espagne, en 1879». Çédille, revista de estudios franceses, 16 [Monografías 9: Diana Cooper-Richet, ed., Regards croisés sur la presse francophone en Espagne et la presse hispanophone en France (XVIII-XX siècles)], 101-114. DOI: https://doi.org/10.25145/j.cedille.2019.17.16.10. 